\title{
Building a Landslide Inventory for West Virginia: Step 1 in Statewide Risk Assessment
}

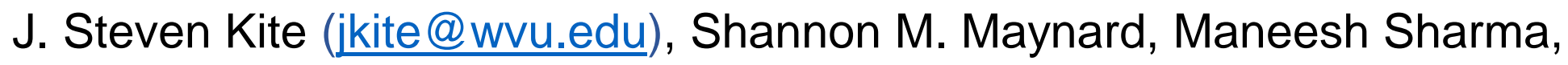
Kurt Donaldson, Matthew Bell, Aaron E. Maxwell, Elizabeth Hanwell, \& Christopher D'Emidio

\section{West Virginia GIS Technical Center (WV GIS TC)}

West Virginia University Department of Geology \& Geography Not a Geological Survey

Session T160: Landslide Inventories, Hazard Assessments, and Risk Reduction Paper 150-2: https://gsa.confex.com/gsa/2019AM/meetingapp.cgi/Paper/337478

1:45 - 2:00 PM, Monday, 23 September 2019 Revised 8 October 2019

224A North Building, Phoenix Convention Center

Yeager Airport, 12 March 2015 Charleston Daily Mail Photo

2018-2021 Effort Funded by FEMA Hazard Mitigation Grant Program \& WV Division of Homeland Security and Emergency Preparedness 
- Landslides = \#2 West Virginia Hazard (FEMA).

- West Virginia $=\mathbf{1 1 . 2 \%}$ of 1973-1983 Landslide Damage in 48 States 。 \#1 in Per Capita Landslide Damage (Brabb, 1984, USGS OF 84-486).

- $\quad 70 \%$ of West Virginia = "High Landslide Incidence"

No Other State $>25 \%$ (USGS PP 1183).

Coterminous

U.S. Landslide Overview Map USGS PP 1183

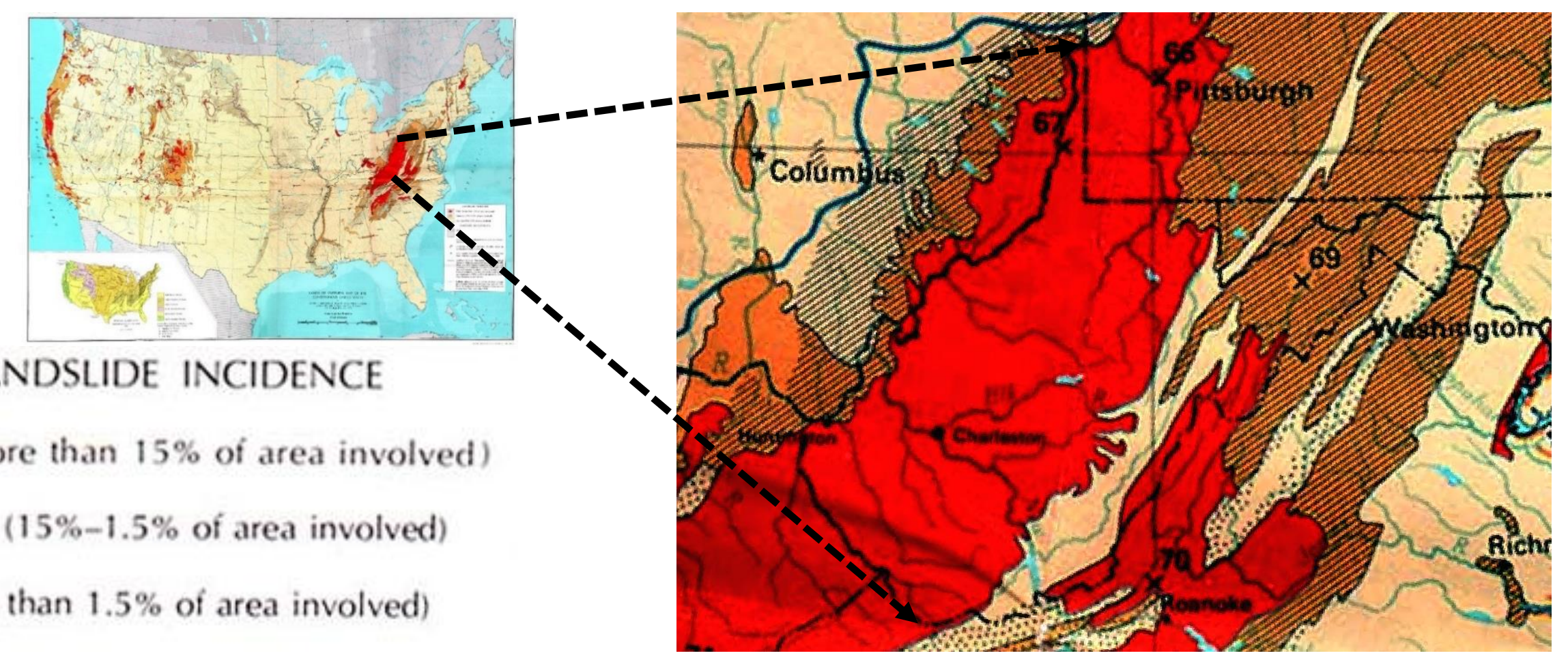




\section{West Virginia Physiography \& NRCS MLRAs}

Existing Physiographic Maps Inadequate for WV Landslide Project

\section{MLRA Boundaries Better}

\section{Provinces \& Subdivisions} Appalachian Plateaus

- Kanawha Section

- Logan Plateau

- Allegheny Mountains Valley \& Ridge

- Ridge \& Valley

- Great Valley Blue Ridge

Red $=$ Highly Landslide-Prone

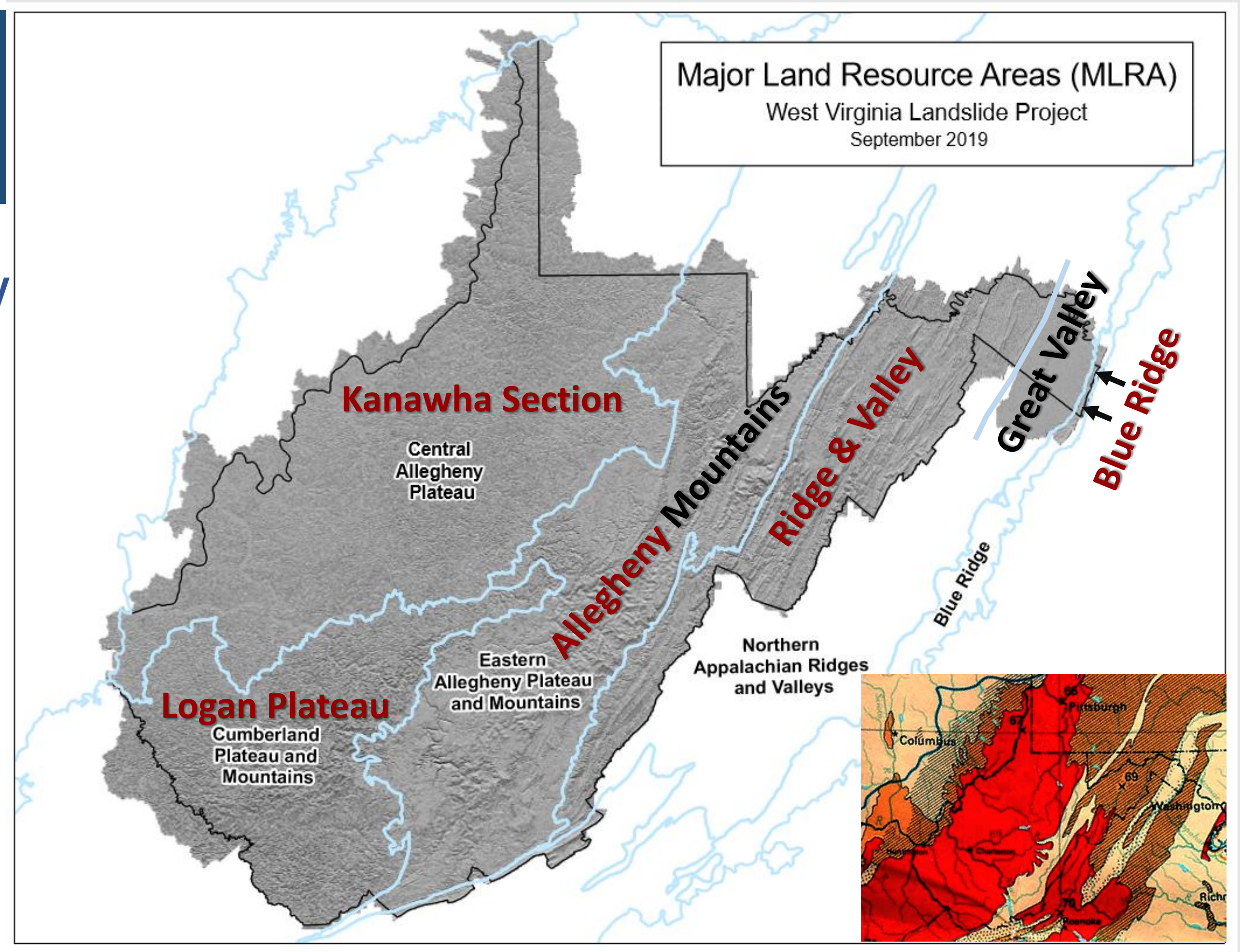




\section{Long-Term Goals:}

- Landslide Inventory (Focus of Talk)

- Step 1A: Compile Statewide Inventory from Pre-Existing Landslide Maps

93,000 Previously Identified Landslides (Revised from > 75,000 in GSA Presentation)

- Disparate Sources: Disparate Landslide Data

\begin{tabular}{|c|c|c|c|}
\hline Purpose & What Was Mapped & Available Tools & Base "Map" \\
\hline Basic Science & Landslide Polygon & Field Work & $1: 62,500$ topo \\
\hline Resource Management & Initiation Point & Image Interpretation & $1: 24,000$ topo \\
\hline Disaster Documentation & Impact Point & GPS & DEMs \\
\hline Site Mitigation & Material \& Mechanics & LiDAR & Road Mileage \\
\hline
\end{tabular}

- Step 1B: New Landslide Mapping from LiDAR-Based DEMs

- Too Many Other Landslide Risk Assessment Goals for a GSA Talk

- Modelling-Based Landslide Susceptibility Maps

- Local Landslide Risk Reports for Hazard Mitigation Planning 
West Virginia

Landslide

\section{Inventory}

\section{Landslide Impact Points}

WV Division of Highways

Landslide Database

1,406 Recent Landslides

Biased to Road Network \& DoH District Involvement

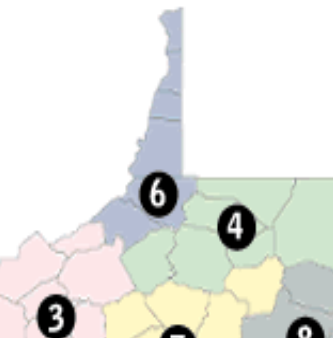

\section{5}

0

(1)

WV: 6th-largest state maintained highway network in US

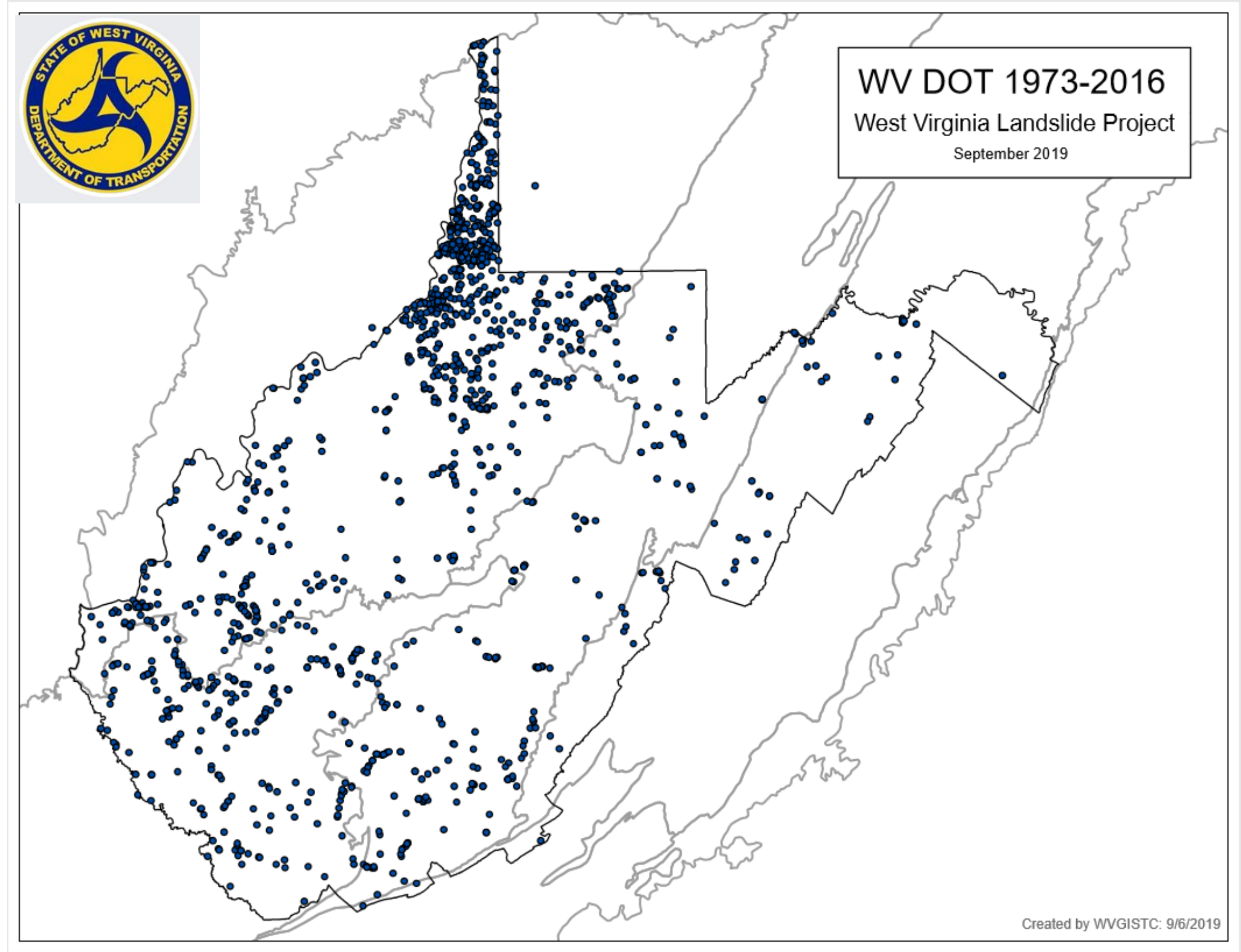




\section{West Virginia}

Landslide

\section{Inventory}

Air-Photo \& Field Based Landslide Polygon Maps

WV Geological \& Economic Survey (Lessing et al.)

39 maps 1:24,000 ( 8 \% of WV)

\section{6,330 Recent \& Older Slides}

If Representative (probably not), 600,000 Landslides Statewide

Biased to Urban Areas with Known Landslide Risk

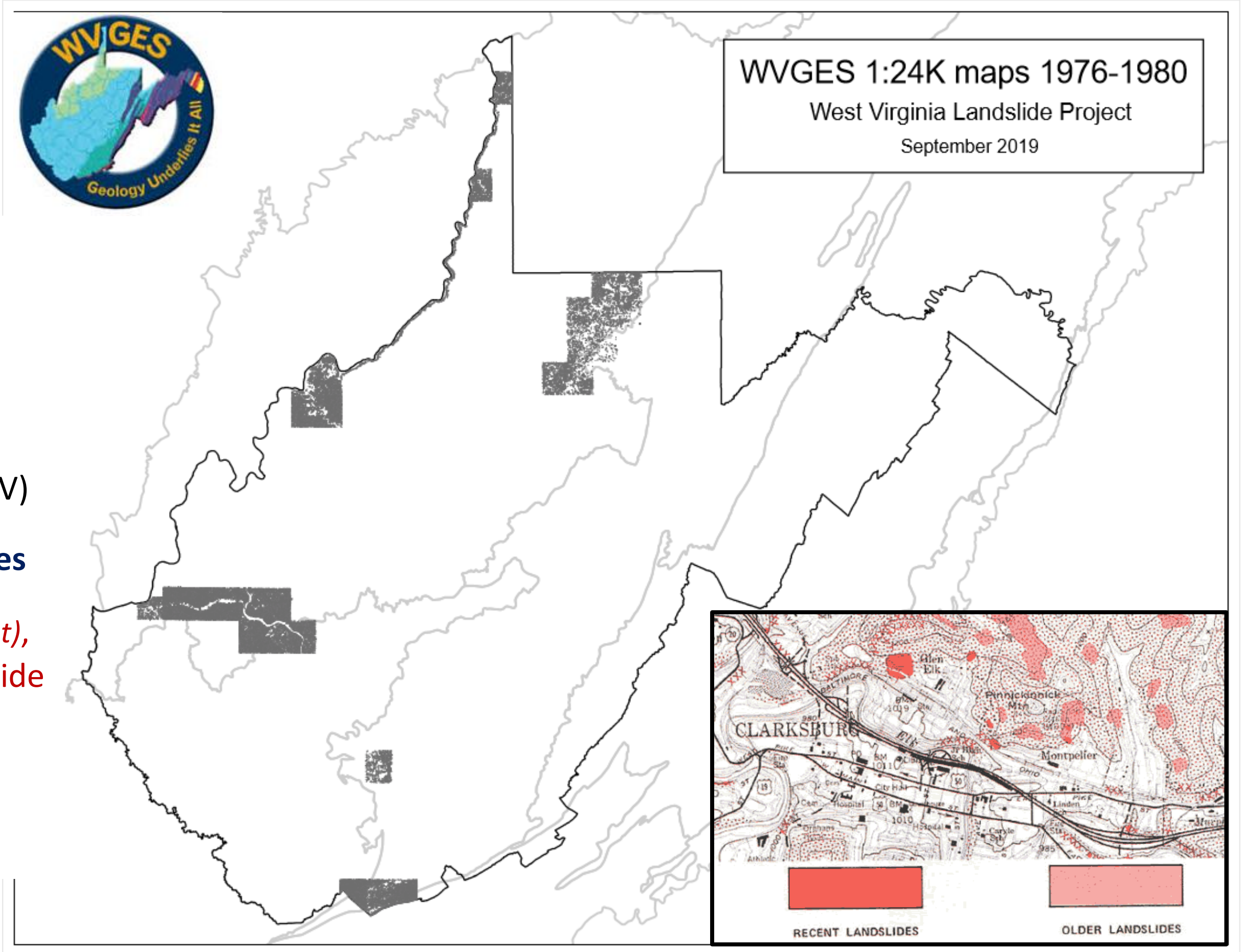


West Virginia

Landslide

\section{Inventory}

\section{Air-Photo \& Field Based Landslide Polygon Maps}

U.S. Geological Survey 19781985 (8 Different Mappers)

382 (of 496) 1:24,000 Quads ( $75 \%$ of WV)

\section{1,307 Active or Recently} Active Landslides

Not Digitized into Inventory:

- Combined Old \& New Landslides

- Old Landslides

- Areas Susceptible to Landsliding

Biased by Varied Mapper Styles \& Varied Land Cover
USGS 1:24K maps 1978-1985

West Virginia Landslide Project

October 2019

Cumberland $1^{\circ} \times 2^{\circ}$ Project Never Públished
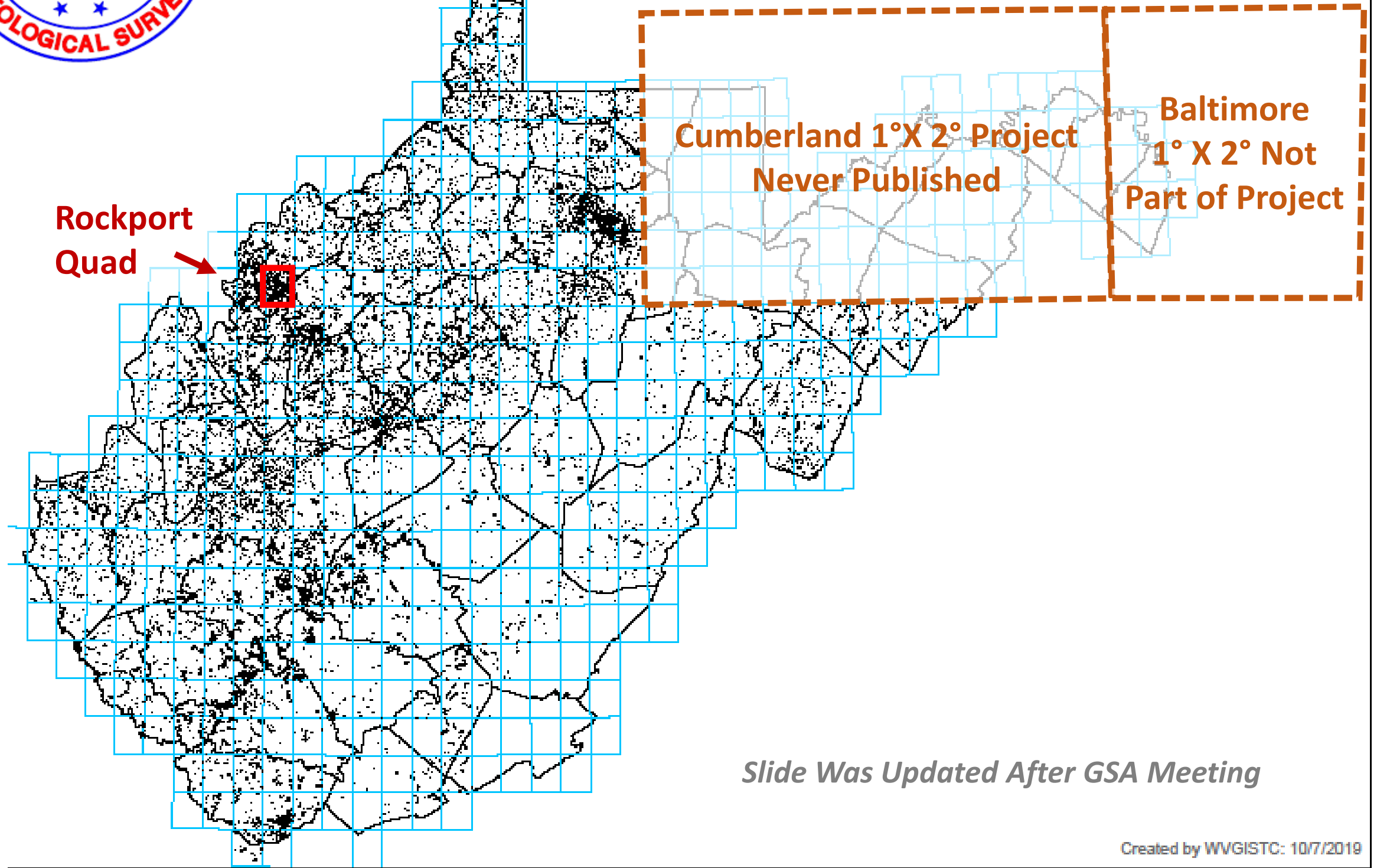

Slide Was Updated After GSA Meeting 


\section{USGS Landslide Mapping 1978-1985}

\section{South Central $1 / 9^{\text {th }}$}

\section{Rockport, WV}

\subsection{Minute Quad}

Hackman, Robert J., and Thomas, Roger E., 1978, Landslides and related features, Ohio, West Virginia, and Pennsylvania; Clarksburg 1 degree by 2 degrees sheet: U.S. Geological Survey Open-File Report OF-78-1056, (1:24,000 scale, 121 maps), https://doi.org/10.3133/ofr781056,

https://ngmdb.usgs.gov/Prodesc/proddesc 54933.htm

Based on B\&W (1960 \& 1976) and IR (1973) Aerial Photos + Field Checked (1976-1977)
ACTIVE OR RECENTLY ACTIVE LANDSLIDE

Complex landslide composed of earthflow, debris slide, earth and rock slump. Identified from other field evidence. Ground extremely unstable sliding accelerated by excavation, loading and changes in drainage conditions. May include be shown separately. Quest ioned where deubtful.

:\% OLD LandsLide

Area of extensive humnocky ground caused by earthflow and earth and rock slump: Lacks clear ovidence of active sliding. Relatively glabelity not affected by small ste, sited in areas away from the edse of ures properly can be reactivated by extensive, rapid excavation, loading, and changes in ground water and surface water conditions. Area of old landslide probably includes recent ones not identified from field evidence or otherwise documented.
Upslope boundary of landslide generally defined upslope boundary of indslide generally defined gradational and not well defined. Questioned where doubtful.

T.+ COMBIHATIOH LaADSLIDE

Area of recent and ord slides in which individut slides are not identified.

SOIL AND ROCK SUSCEPTIBLE tO LANOSLIDING Soil and rock similar to that involved in landslides el sewhere in map area; primarily areas associated with other rock types. Bock weathers rapidly on exposure forning clayey soil highly susceptible to sliding. Includes coves (U-shaped shallow valleys) containino thick layers of clayey soil that are very susceptible to sliding where excavation breaks continuity of slope and where overloaded by artificial fill.

AREAS LEAST PRONE TO LANDSLIDES

Map areas in which no patte primarily valley floors, ridge tops and broad benches; modification by excavation and fill may lead to local landslides.

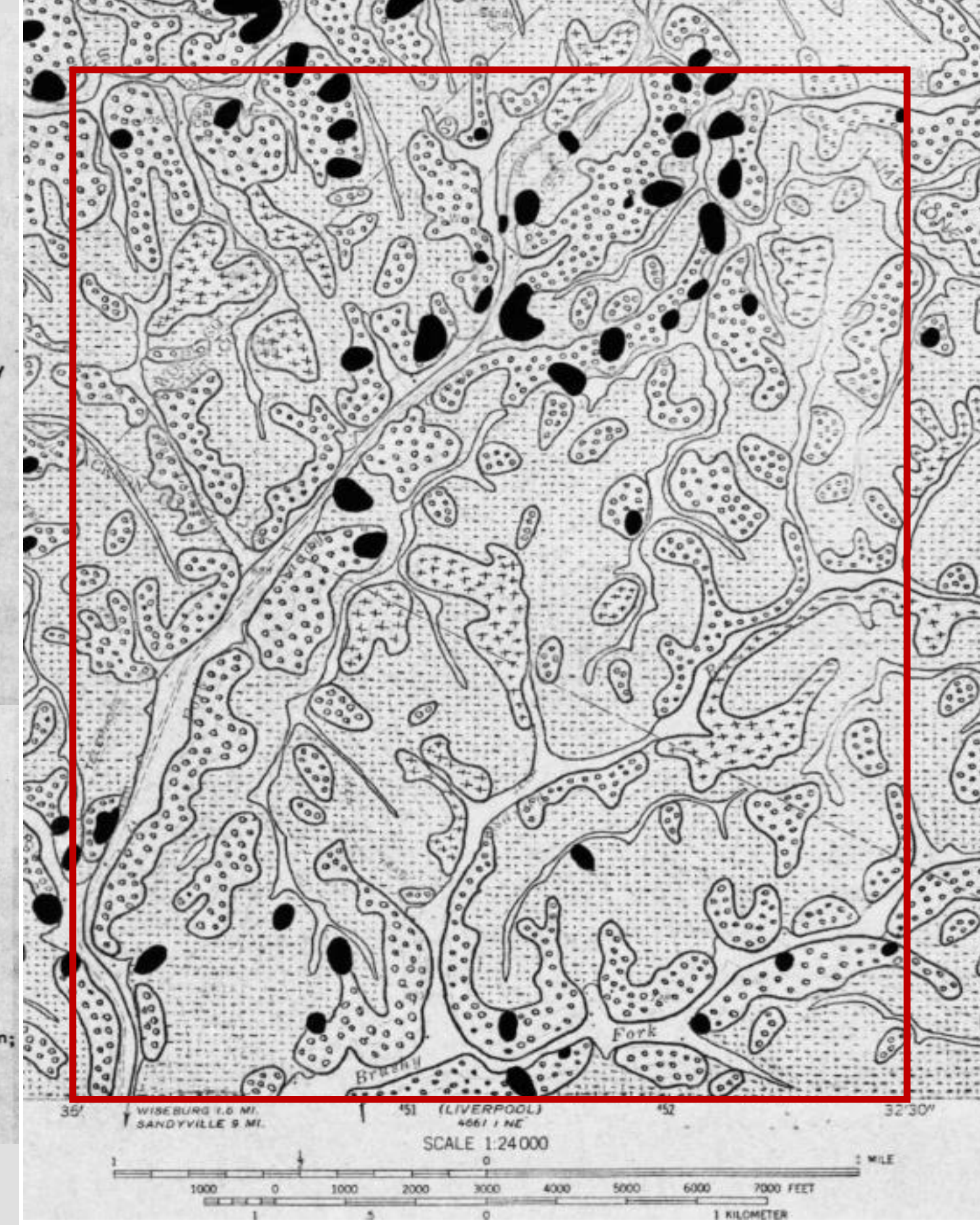




\section{USGS Landslide} Mapping 1978-1985

\section{South Central $1 / 9^{\text {th }}$ Rockport, WV 7.5 Minute Quad}

Hackman, Robert J., and Thomas, Roger E., 1978, Landslides and related features, Ohio, West Virginia, and Pennsylvania; Clarksburg 1 degree by 2 degrees sheet: U.S. Geological Survey Open-File Report OF-78-1056, (1:24,000 scale, 121 maps), https://doi.org/10.3133/ofr781056, https://ngmdb.usgs.gov/Prodesc/proddesc 54933.htm

Based on B\&W (1960 \& 1976) and IR (1973) Aerial Photos + Field Checked (1976-1977)

Biased by a 495 mm (19 1/2 inch) 2 hr 10 min Rainfall, 18 Jul 1889
Active or Recently Active Landslide: 47 small polygons - Only Landslides in WV GIS TC Inventory (Sept 2019)

(0) Old Landslide: 88 large polygons - Not in Inventory

Combination (Old \& New) Landslide: 12 large polygons - Not in Inventory

Soil \& Rock Susceptible to Landsliding: huge polygons covering $\sim$ half of area - Not in Inventory

Areas Least Susceptible to Landsliding: thin polygons floodplains \& ridge crests

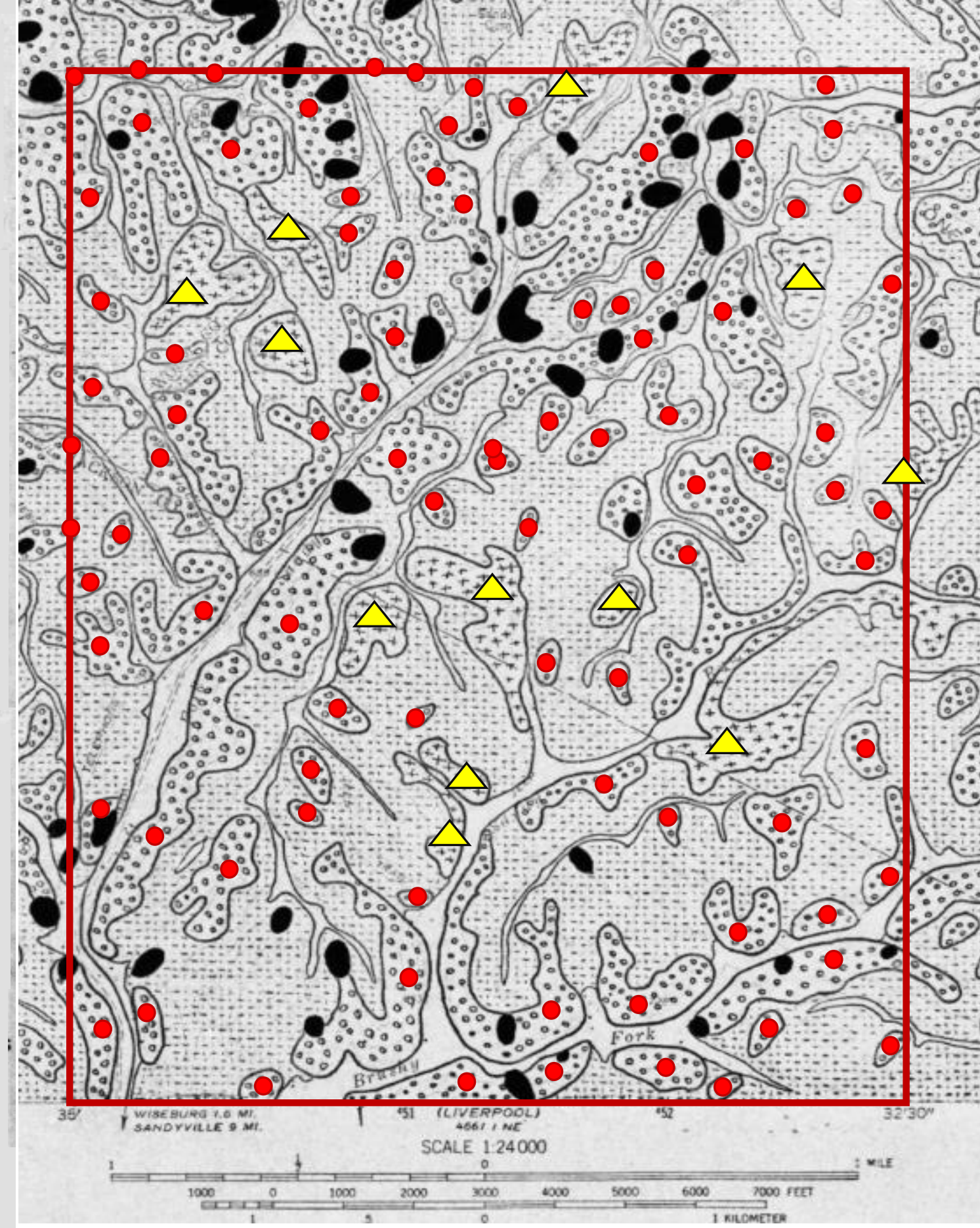




\section{USGS Landslide} Mapping 1978-1985

\section{South Central $1 / 9^{\text {th }}$}

\section{Rockport, WV}

\subsection{Minute Quad}

Hackman, Robert J., and Thomas,

Roger E., 1978, Landslides and related features, Ohio, West Virginia, and Pennsylvania; Clarksburg 1 degree by 2 degrees sheet: U.S. Geological Survey Open-File Report OF-78-1056, (1:24,000 scale, 121 maps) https://doi.org/10.3133/ofr781056, https://ngmdb.usgs.gov/Prodesc/proddesc 54933.htm

\section{Implications:}

- Inventory is Missing >2/3 of USGS Slides

- If 147 Slides per $1 / 9^{\text {th }}$ Quad Is Typical (Probably Not), WV Could Have $>600,000$ Slides!
Active or Recently Active Landslide: 47 small polygons - Only Landslides in WV GIS TC Inventory (Sept 2019)

Old Landslide: 88 Large Polygons - Not in Inventory

Combination (Old \& New) Landslide: 12 Large Polygons - Not in Inventory

Soil \& Rock Susceptible to Landsliding: Huge Polygons Covering Half of Area - Not in Inventory

Areas Least Susceptible to Landsliding: Not Much! Floodplains \& Ridge Crests

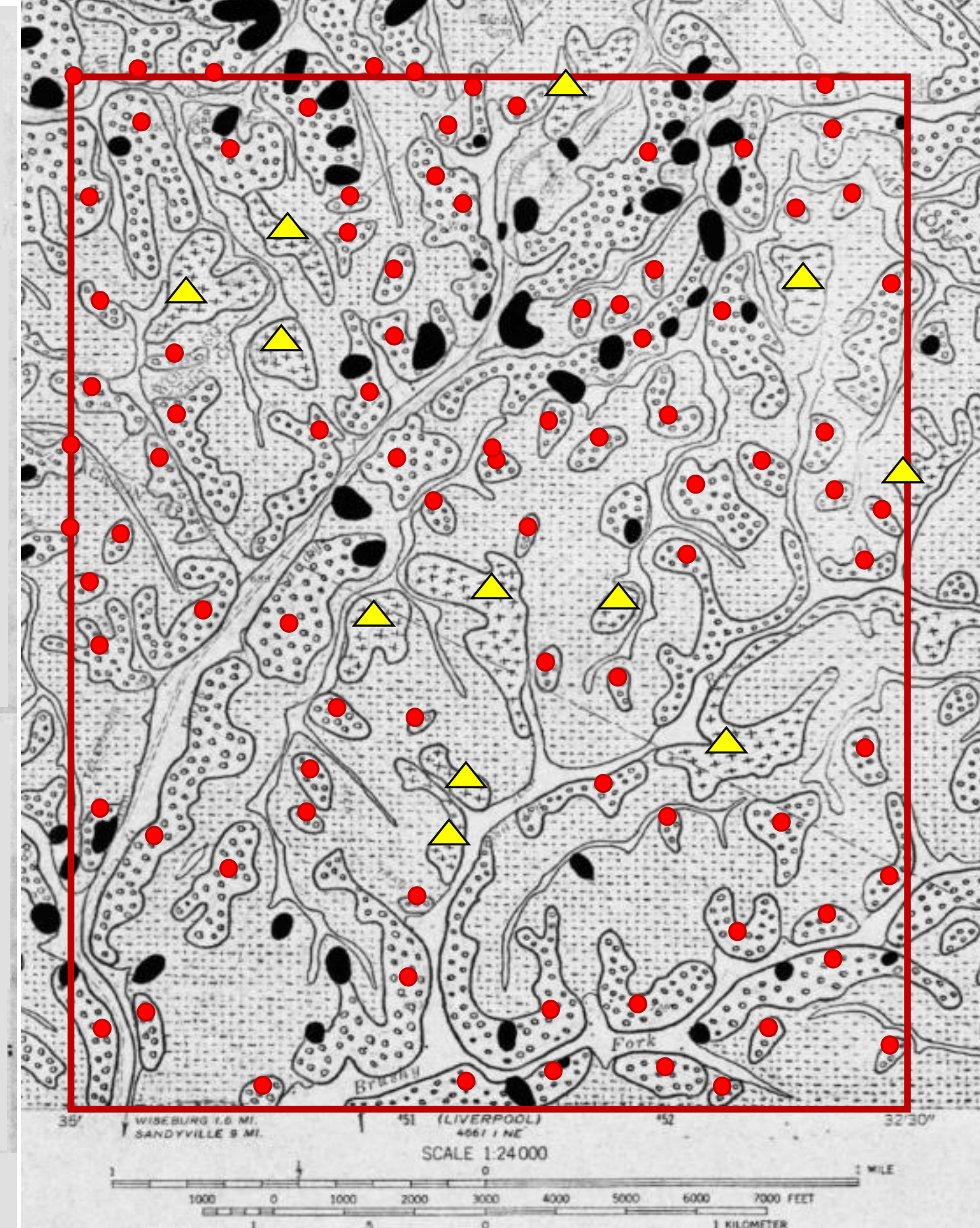


Other Landslide

\section{Inventory Data}

Field Mapped Polygons

Landers \& Smosna WVGES (Fatal 1973 Debris Flows)

Air-Photo \& Field Based Landslide Initiation Points Jacobson et al. USGS (GIS Mapping 1985 "Flood")

Schneider (1973 UT-K PhD on 1969 "Debris Slides")

DEM Based Landslide Polygons Konsoer \& Downing (2008 WVU MS Theses-USGS EDMAP) Yates \& Kite, NPS 1:10,000 Mapping $(2014,2016)$

Other Sources We Could Add Geotechnical Projects, Media Reports, Landslide Reporting App
How Do We Best Fit Different Data Types Different Purposes Different Researchers Different Methods to a Single Database?

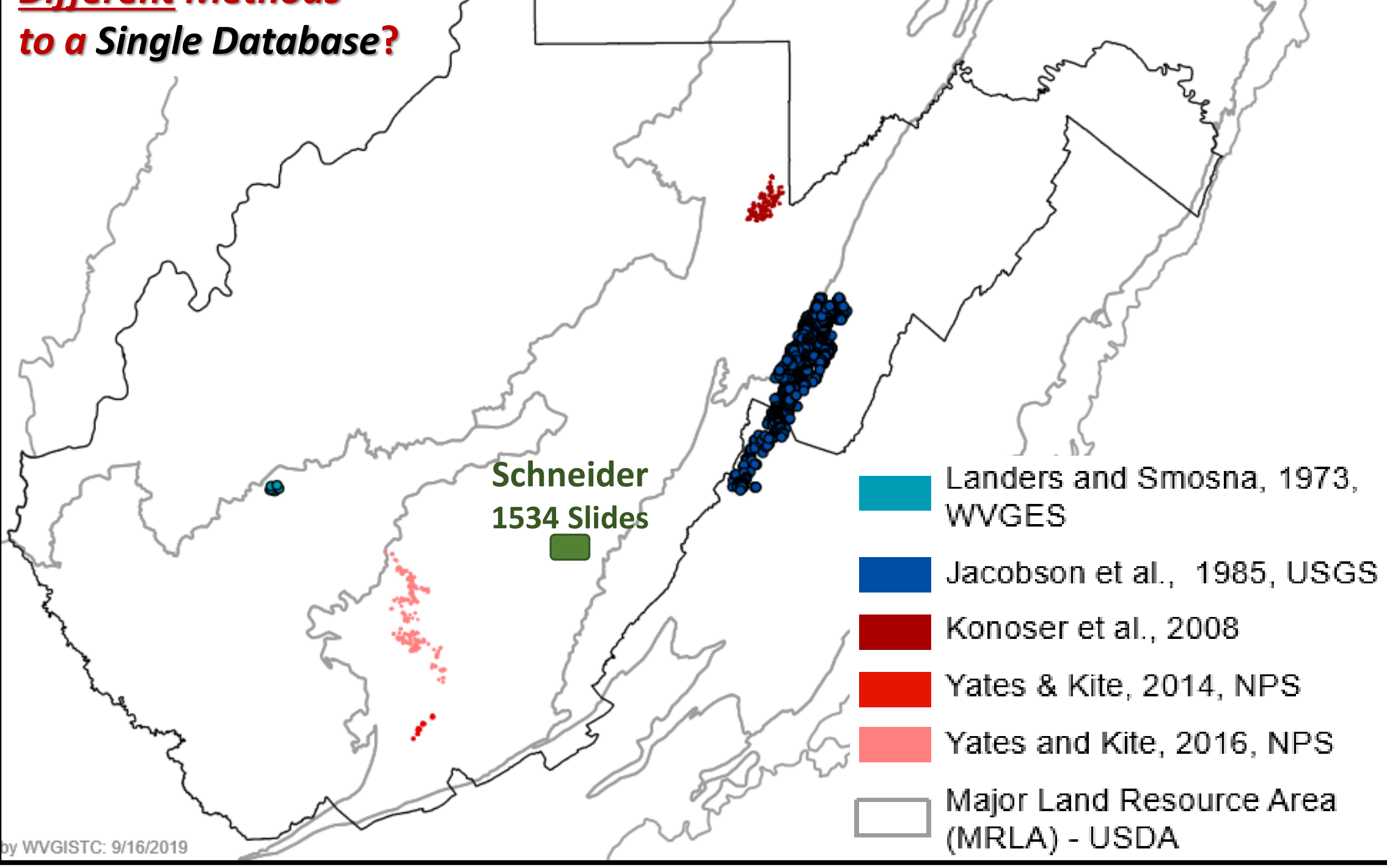




\section{West Virginia Landslide Mapping}

\section{New Landslide Mapping by WV GIS TC}

- Landslide Mapping Protocol for QL2 LIDAR 1 m DEMs

- Hill-Shade Base Map Layer (Best Available Statewide)

- Slope-Shade Layers (1 m DEM Areas Only )

- LiDAR Is Crucial

- Good Coverage in Eastern West Virginia

- Update as QL2 1 m DEMs Arrive

- QL2 1 m DEMs for All WV Unlikely by June 2021

- May Map on QL3/QL4 2 m DEMs Where QL2 LiDAR is Unlikely before Project Ends

- Many Areas Only 3 m DEMs - Worthless for Mapping

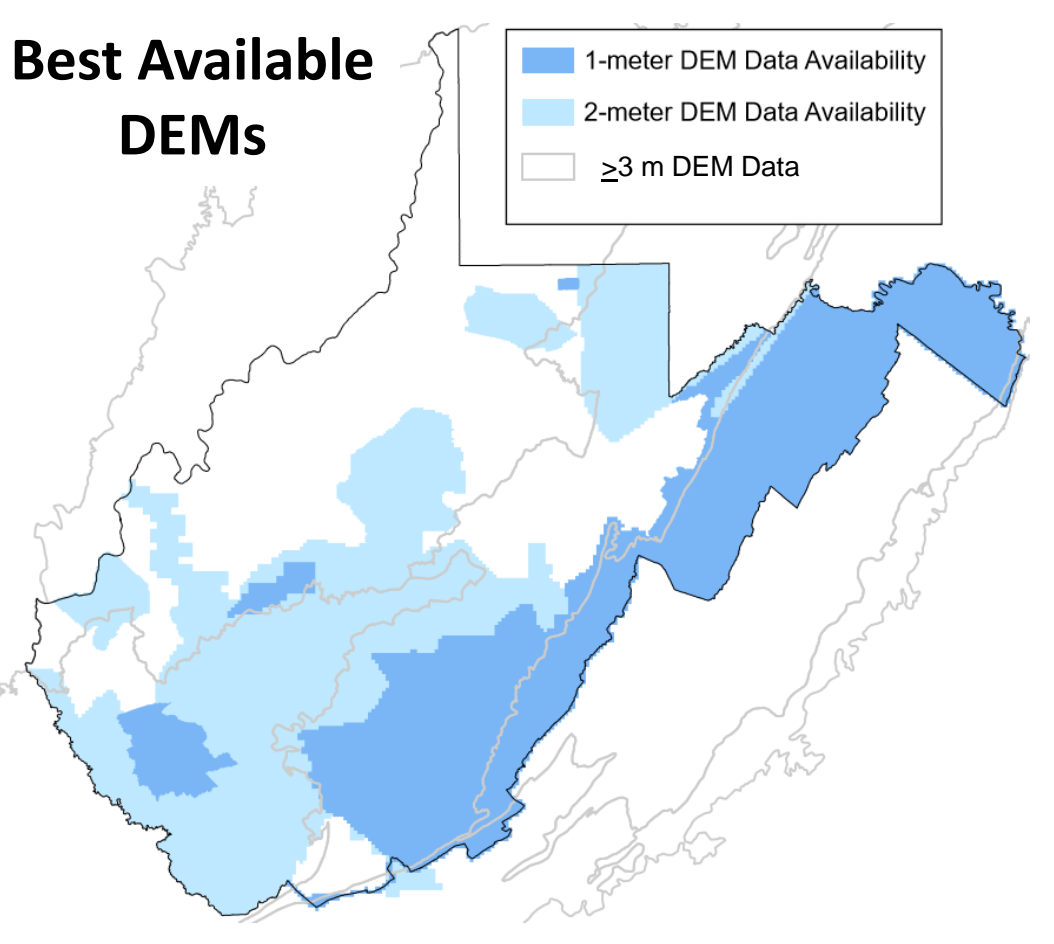

Find Best-Available Elevation Sources for West Virginia at www.mapwv.gov/floodtest/docs/

WV_FloodTool_ElevationSource_Metadata.pdf 


\section{Reality Check After Mapping Trials - Revised 4 June 2019}

\begin{tabular}{|c|c|c|c|}
\hline & & \multicolumn{2}{|c|}{ Material before Failure (Engineering Criteria) } \\
\hline \multicolumn{2}{|c|}{ Failure Motion } & Bedrock & Soil \\
\hline General Motion & Type of Motion & Rock & Debris = Default Material in WV \\
\hline \multirow{3}{*}{ Falling } & Fall & \multirow{2}{*}{ Rock Fall } & \multirow{2}{*}{ Rare \& Difficult to Discern } \\
\hline & Topple (Falling Over) & & \\
\hline & $\begin{array}{l}\text { Collapse * (Roof Failure } \\
\text { into Large Void Space) }\end{array}$ & \multicolumn{2}{|c|}{ Karst \& Mine Subsidence Outside Project Scope } \\
\hline \multirow{2}{*}{ Sliding } & $\begin{array}{l}\text { Slide (Translational Sliding } \\
\text { along Planar Surface) }\end{array}$ & \multirow{2}{*}{\multicolumn{2}{|c|}{ Slide }} \\
\hline & $\begin{array}{l}\text { Slump (Rotational Sliding } \\
\text { along Concave Surface) }\end{array}$ & & \\
\hline \multirow{4}{*}{ Flowing } & Lateral Spread & Lateral Spread & Rare \& Difficult to Discern \\
\hline & $\begin{array}{l}\text { Creep *(Slow Downslope } \\
\text { Creep with No Slip Su rface) }\end{array}$ & \multicolumn{2}{|c|}{ Creep Not Discernable from Stable Slope } \\
\hline & $\begin{array}{l}\text { Flow (Rapid Viscous } \\
\text { Channel Flow) }\end{array}$ & Very Rare & Debris Flow \\
\hline & $\begin{array}{l}\text { Avalanche (Very Rapid } \\
\text { Flow over Compressed Air) }\end{array}$ & \multicolumn{2}{|c|}{ True Avalanches Are Very Rare in Central Appalachians } \\
\hline Complex & $\begin{array}{l}\text { Multiple Motion Types - } \\
\text { e.g. Multiple Small Failures }\end{array}$ & \multicolumn{2}{|r|}{ Multiple Failures } \\
\hline Undifferentiated & $\begin{array}{l}\text { Failure Process Unclear } \\
\text { from Available Data }\end{array}$ & \multicolumn{2}{|c|}{ Undifferentiated Slope Failure } \\
\hline
\end{tabular}

Landslide Identification

Biased to Head Scarp

Morphology.

- DEMs Will Not Allow

Differentiation of Debris vs.

Earth or Soil vs. Rock.

- Consistent Translational Slide

vs. Rotational Slump

Differentiation Is Unlikely. 


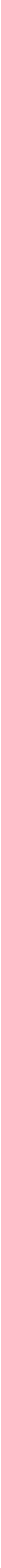




\section{WV GIS TC}

\section{Landslide Mapping} March-September 2019

\section{WV GIS TC Mapping on} LiDAR-Based DEMs

\section{8,991 Failures ( $\geq 10 \mathrm{~m}$ wide)} Most from $1 \mathrm{~m}$ DEMs

- 334 Debris Flows

- 241 Lateral Spreads

- 8,416 Other Failures $>97$ \% "Slides" (or Slumps)

Few Rock Falls Identified

\section{Mapped Landslides} Verified on 2 m DEMs

- 1,082 WVGES (1976-80) Monongalia Co. Slides

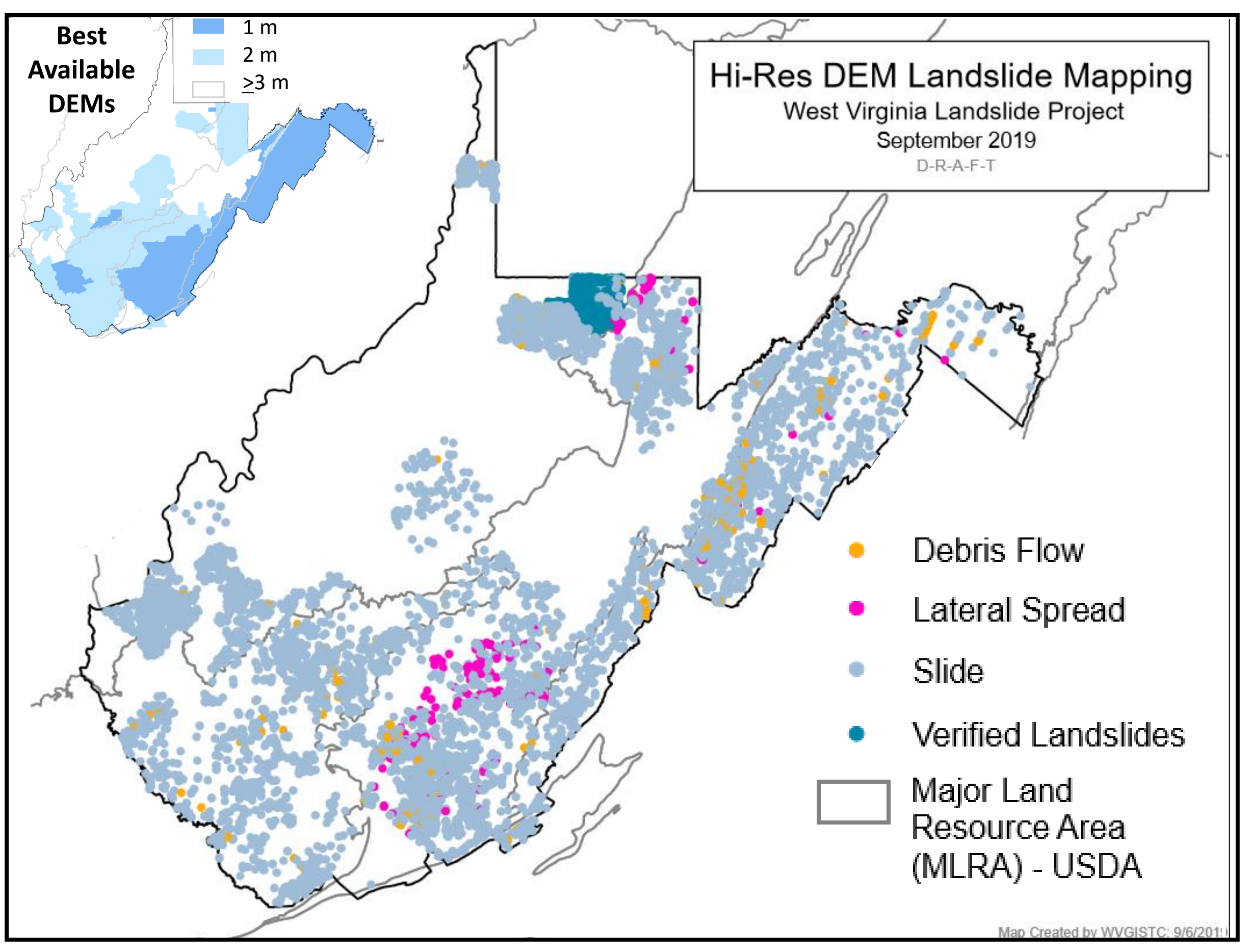




\section{WV GIS TC West Virginia Landslide Mapping}

- Newly Mapped Landslides on LiDAR-Based DEMs Plotted vs. USGS PP 1183 Landslide Overview Map
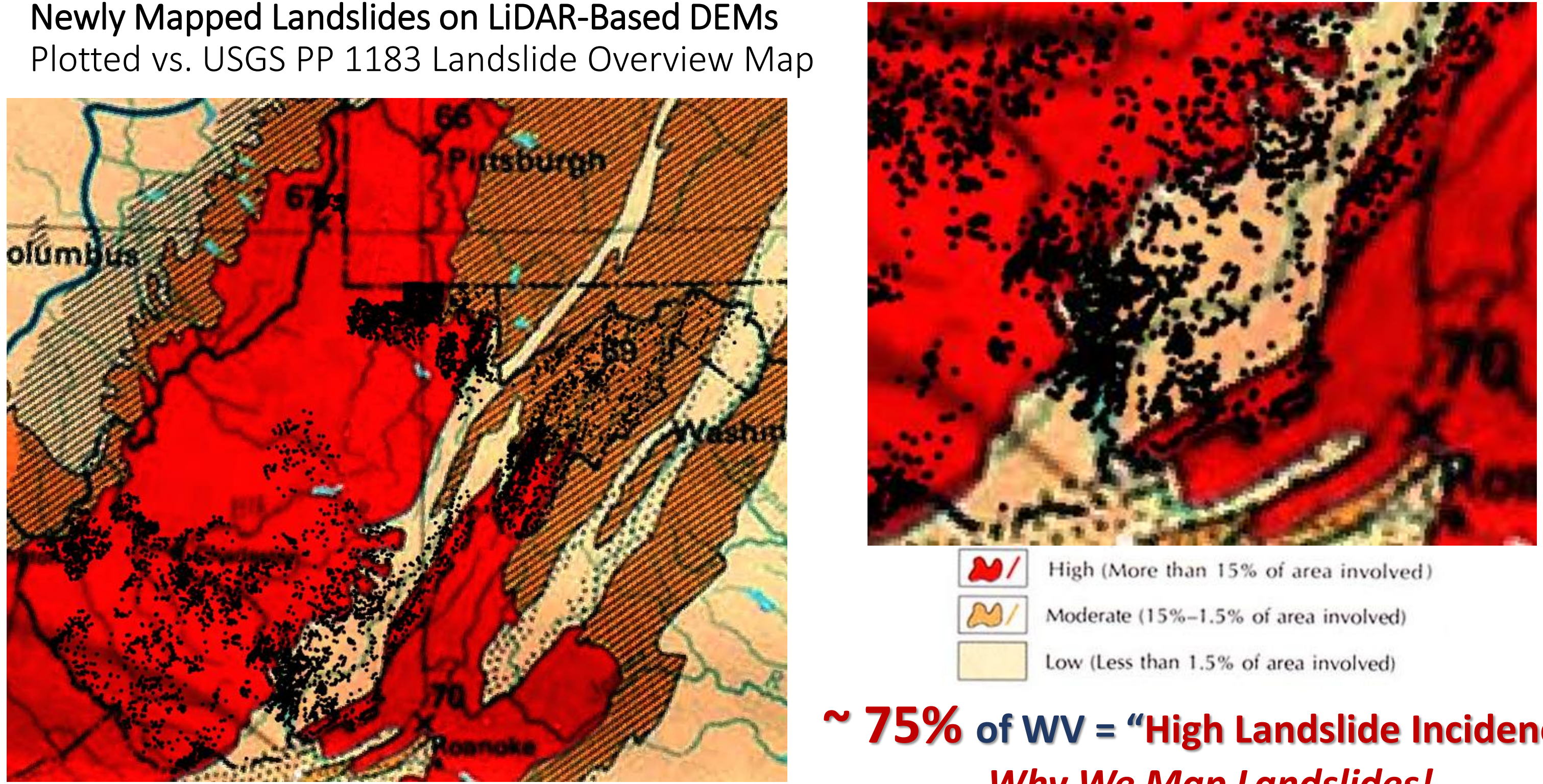

75\% of WV = "High Landslide Incidence" Why We Map Landslides! 